\title{
Electroencephalography as a diagnostic technique for canine neurological diseases
}

\author{
Marcin Wrzosek \\ Department of Internal Medicine and Clinic of Horses, Dogs and Cats, Faculty of Veterinary Medicine, \\ Wroclaw University of Environmental and Life Sciences, 50-366 Wroclaw, Poland \\ marcin.wrzosek@up.wroc.pl
}

Received: November 16, 2015 Accepted: May, 11, 2016

\begin{abstract}
Electroencephalography (EEG) is a non-invasive examination method for the assessment of functional central nervous system (CNS) disturbances. In human medicine it has a special importance as a diagnostic tool for epilepsy. Although many studies were done on the use of EEG for diagnostics of canine central nervous system disorders, the technique is still not applied routinely. The purpose of this paper was to review the use of the electroencephalography in canine neurological disorders of central nervous system diagnosis and assess the future perspectives of this technique in veterinary medicine.
\end{abstract}

Keywords: dog, central nervous system disorders, electroencephalography, review.

\section{Introduction}

Electroencephalography (EEG) is a useful and noninvasive examination method for the diagnosis of functional central nervous system (CNS) disturbances, and has a special importance as a routine diagnostic tool for epilepsy in humans $(13,28)$. When recording the electrical activity of the cortex, EEG is extremely sensitive to any other electrical activity, including muscle, cardiovascular, and ocular activity of the patient. It is therefore very demanding with respect to a patient's cooperation and patience. This fact causes difficulties regarding its use in animal assessment. Nevertheless, EEG has been used in animal models of human epilepsy and for the differentiation of central nervous system diseases in veterinary medicine $(3,9,19,29,32,40,44)$.

Although canine epilepsy is the most common neurological disturbance in veterinary medicine (33), EEG is still not used as a routine examination in assessment of those patients. Different techniques of the EEG examination on both awake and sedated dogs with various anaesthetic agents have been reported $(18,21)$. It appears that various sedatives change the EEG appearance, but still there is no uniform standard protocol that could be used for canine central nervous system examination $(2,4,8,17,18,34)$. In most of the latest publications, propofol alone, medetomidine and propofol, or medetomidine alone, with or without muscle relaxants, have been proposed as appropriate for use in canine EEG (Table 1), (4, 7, 23).

History of electroencephalography (EEG). In 1929, German psychiatrist Hans Berger performed the first single channel tracing EEG recording, showing that the electricity of the human brain could be recorded (5). He reported changes in EEG with processes such as eye opening and closing and active thinking. The recording, originally called the "Berger rhythm", then the $\alpha$ rhythm, is an $8-12 / \mathrm{s}$ posterior maximal rhythm that occurs when the subject is in a restful, not asleep state. The multichannel EEG recordings came into play in the 1930s, when an EEG recording associated with epilepsy, including spikes and waves, was described (12). From the 1930 s to the 1970 s, the EEG served as a noninvasive study to assist with localisation of cerebral dysfunction. It was always used by most experts in its interpretation as an adjunct to the history and neurological examination. In these times EEG coexisted with invasive CNS tests like angiography and pneumoencephalography, but with the arrival of the CT scan, and later the MRI, the EEG lost its primacy as a test to localise structural abnormalities; however, it still has several important indications (28). 
Table 1. Comparison of various EEG electrodes and montages reported in dogs. SNE - subdermal needle electrodes, SWE - subdermal wire electrodes, IE - idiopathic epilepsy, SE - secondary epilepsy, derivation/electrodes abbreviations: even: right side, uneven: left side, F- frontal, T - temporal, C - central, $\mathrm{P}$ - parietal, O - occipital, PD- paroxysmal discharges

\begin{tabular}{|c|c|c|c|c|}
\hline Author & Electrode type & Montage & Sedation protocol & $\begin{array}{l}\text { Amount of PD in } \\
\text { epileptic dogs }\end{array}$ \\
\hline Brauer et al. (6) & SNE, $12 \mathrm{~mm}$ & $\begin{array}{l}\text { Five electrodes (F3, } \\
\mathrm{F} 4, \mathrm{Cz}, \mathrm{O} 1, \mathrm{O} 2)\end{array}$ & $\begin{array}{l}\text { Propofol } 7.5 \mathrm{mg} / \mathrm{kg} \text { iv, then CRI } 0.37 \\
\mathrm{mg} / \mathrm{kg} / \mathrm{min} \text { Rocuronium bromide } 0.4 \\
\mathrm{mg} / \mathrm{kg} \text { iv, photic stimulation, } \\
\text { hyperventilation }\end{array}$ & $25 \% \mathrm{IE}, 29 \% \mathrm{SE}$ \\
\hline Davis et al. (10) & Corticogram & $\begin{array}{l}16 \text { channel, scalp } \\
\text { recording }\end{array}$ & no sedatives, continuous recording & $100 \%(n=6 / 6)$ \\
\hline Jeserevics et al. (23) & $\begin{array}{l}\text { SNE 30-gauge, } 15 \\
\text { mm (monopolar } \\
\text { stainless-steel needle } \\
\text { electrodes) }\end{array}$ & $\begin{array}{l}14 \text { channel ref } \\
\text { montage (F7, F3, F4, } \\
\text { F8, T3, C3, Cz, C4, } \\
\text { T4, P3, Pz, P4, O1, } \\
\text { O2), bipolar }\end{array}$ & $\begin{array}{l}\text { Medetomidine } 40-60 \mu \mathrm{g} / \mathrm{kg} \mathrm{IM} \text {, } \\
\text { additional } 10-20 \mu \mathrm{g} / \mathrm{kg} \text { in not ready for } \\
\text { manipulations after } 20-30 \mathrm{~min}\end{array}$ & $20 \%(\mathrm{n}=3 / 15)$ \\
\hline $\begin{array}{l}\text { Pellegrino and Sica, } \\
\text { (36) }\end{array}$ & SNE, $15 \mathrm{~mm}$ & $\begin{array}{l}8 \text { electrodes }(\mathrm{F} 3, \mathrm{~F} 4 \\
\mathrm{P} 3, \mathrm{Pz}, \mathrm{P} 4, \mathrm{O} 1, \mathrm{Oz} \\
\mathrm{O} 2), \text { bipolar }\end{array}$ & Xylazine SC $1 \mathrm{mg} / \mathrm{kg}$ & $55 \%(\mathrm{n}=5 / 9)$ \\
\hline Morita et al. (30) & subcutaneous & $\begin{array}{l}12 \text { channels, mono-, } \\
\text { bipolar }\end{array}$ & Xylazine & $100 \%(\mathrm{n}=9 / 9)$ \\
\hline Berendt et al. (2) & subcutaneous & 14 channels & Acepromazine, pethidine & $65 \%(n=15 / 23)$ \\
\hline $\begin{array}{l}\text { Jaggy and } \\
\text { Bernardini (19) }\end{array}$ & $\begin{array}{l}\text { SNE (subdermal } \\
\text { platinum needle) } 12 \\
\text { mm }\end{array}$ & $\begin{array}{l}8 \text { channel, mono-, } \\
\text { bipolar }\end{array}$ & $\begin{array}{l}\text { Medetomidine IV } 0,025 \mathrm{mg} / \mathrm{kg} \\
\text { Propofol bolus } 2 \mathrm{mg} / \mathrm{kg} \text { IV, then CRI } \\
0.05-0.1 \mathrm{mg} / \mathrm{kg} / \mathrm{min} \text { Atipamezole } \\
0.125 \mathrm{mg} / \mathrm{kg} \mathrm{IV}\end{array}$ & $86 \%(n=32 / 37)$ \\
\hline Holliday et al. (16) & subcutaneous & 6 channels & Chlorpromazine, photic stimulation & $61 \%(\mathrm{n}=43 / 70)$ \\
\hline $\begin{array}{l}\text { Klemm and Hall, } \\
\text { (26) }\end{array}$ & subcutaneous & 8 channels & Pentobarbital, thiopental & $99 \%(\mathrm{n}=90 / 91)$ \\
\hline
\end{tabular}

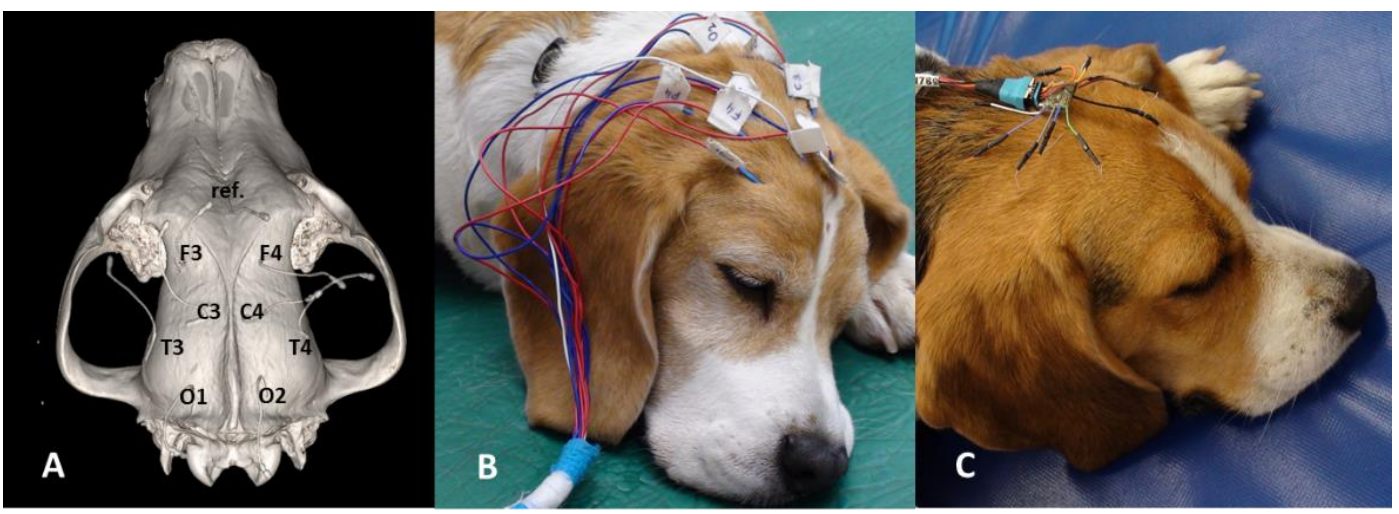

Fig. 1. (A) Computed tomography 3D reconstruction of a canine skull with an example of 8-channel montage electrodes localisation. (B) Localisation of the SNE (subdermal needle electrodes) (C) subdermal wire electrodes (SWE) for 8-channel montage. Monopolar montage: F3, F4, C3, C4, T3, T4, O1, and O2, (F - frontal; T - temporal; C - central; $\mathrm{O}$ - occipital; odd numbers = left hemisphere; even numbers = right hemisphere)

Neural generators of the EEG. Electroencephalograph records spontaneous cortical electric function, registers the activity of the frequency $(0.5-90 \mathrm{~Hz})$ and amplitude (1-500 microvolt) that is adequate for cortical activity. The recording mainly comes from three sources: synaptic activity of cortical neurons, changes of action potentials at the glial cells surface, and summarised electrical activity generated by large populations of neurons, mainly from cortical neuronal layers $(12,20,41)$.

Technical requirements. The EEG recording is performed by electrodes which are positioned on the skull surface. There is a variety of electrodes that have been used in dog studies (Table 1). Standard electrodes for the EEG recording in humans are cup-shaped discs which are glued to the head skin surface. The hair of the examined patient should be shaved. The goal of the electrode placing is to obtain the resistance between the electrode and the skin lower than 1-5 ohms (permissible $<10 \mathrm{ohms}$ ), in order to receive appropriate recording parameters. Due to the practical reasons, to obtain excellent conductivity (with resistance lower that $5-10 \mathrm{ohms}$ ) in most cases, the use of subdermal needle electrodes (SNE): cadmium (38), platinum (15), $\mathrm{Ag}-\mathrm{AgCl}$ (27), steel (6), is recommended. In some exceptions cup-shaped electrodes were used (9), but they are of low practical value. Recently, new promising subdermal wire electrodes (SWE) have been introduced in dog EEG (21). The specific order of electrode pairs is called a montage (12). Electrodes are named after the nomenclature used in human electroencephalography as F - frontal, C - central, $\mathrm{T}$ - temporal, $\mathrm{P}$ - parietal, $\mathrm{O}$ - occipital, and numbered with odd numbers on the right side and even on the left side (Fig. 1). Various montages of electrodes on the dog's head were proposed; all were adapted from the human 10-20 montages (Figs 1, 2). In dogs, monopolar montage is the most often used, comprised of 8-channel 
single-pole assembly (F3, F4, C3, C4, T3, T4, O1, O2, -Ref., ECG-Ref., Resp-Ref.). The reference electrode is placed on the vertex, nose, or neck (4) and ground is usually placed on the neck. In some cases, a bipolar assembly (montage) is used (F3-C3, C3-T3, T3-O1, F4T4, C4-T4, T4-O2 (Figs 1, 2). The EEG examination settings can be used as follows: transverse pages $30 \mathrm{~mm} / \mathrm{s}, 70 \mu \mathrm{V} / \mathrm{cm}$ amplitude, $30.0 \mathrm{~Hz}$ high-pass filter (HF.), $0.016 \mathrm{~Hz}$ low-pass filter (Lf.), and $50.0 \mathrm{~Hz}$ notch filter. During the EEG recording all data are stored in an acquisition station which allows later detailed analyses.

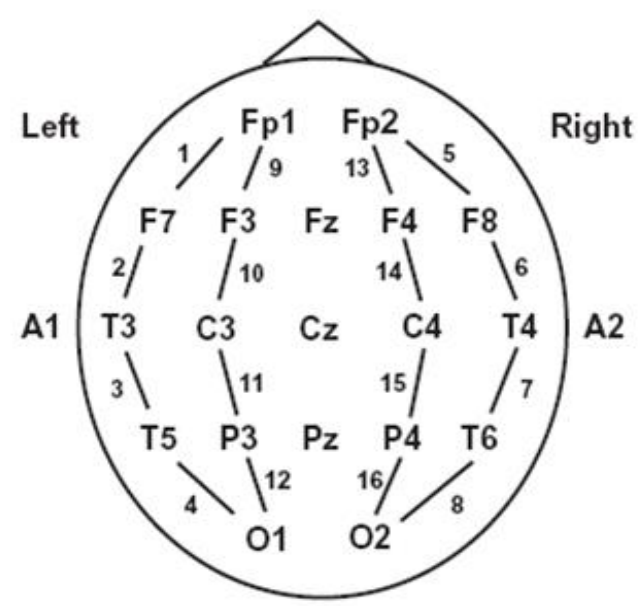

Fig. 2. A standard 10-20 EEG electrode localisation on the scalp, with longitudinal montage (so called "double banana") used in human examination

Activation techniques. Activation procedures are often used in human EEG examination to bring out the higher amount of abnormalities (20). The routine activation procedures are photic stimulation, hyperventilation, and recording during drowsiness and sleep, or sleep deprivation (42), eye closure, mental activity or anti-epileptic drug (AED) withdrawal (28). In canine studies photic stimulation and hyperventilation have been described $(6,45)$. Photic stimulation is based on flashing very bright lights close to the patient, usually through closed eyelids. The frequency varies from 1 to 20 flashes/s. Normal response involves either no response or occipital driving to the light flashes. In humans abnormalities during photic stimulation (11) consist of a markedly asymmetric occipital driving response (abnormal on the side lacking the response) or spikes (22). In dogs this procedure, so far, has not shown a significant increase in pathological response (6). The light flash is usually indicated by a photic monitor in one of the EEG channels (Fig. 3). Hyperventilation may emphasise focal slowing or bring out interictal spikes or spike waves in individuals with absence seizures (35), and can be useful for precipitating partial or non-epileptic seizures as well (1). A human patient is asked to breathe deeply and rapidly for up to $4 \mathrm{~min}$. In canine patients, hyperventilation was performed by passive ventilation in intubated dogs under general anaesthesia.
This technique applied in dogs still did not significantly promote the amount of superimposed transients (SIT) (6). In humans, it is also normal to show no EEG change with hyperventilation (1). Sleep and sleep deprivation are not used as activation procedures in veterinary medicine due to ethical reasons. In humans, drowsiness is the time of the most intensive EEG changes observed, but also the most dangerous in terms of producing and misinterpreting normal variant EEG patterns like k-complexes or sleep spindles (12). As canine EEG is performed in sedation, such activation techniques as volitional eye closure and provocation of mental activity are not possible.

Features of EEG. Electroencephalogram may be evaluated visually or quantitatively, by spectral analysis (quantitative EEG, q-EEG). Two activities are analysed by visual evaluation: background activity (BGA) and SIT (Fig. 4). BGA is a setting in which a given normal or abnormal pattern appears and from which such pattern is distinguished. SIT is a phenomenon with abrupt onset, rapid attainment of maximum, and sudden termination (28). Both can be physiological and pathological. Spectral analysis is used to evaluate the relative and direct values. Those objective values are the mathematical presentation of electroencephalogram, upon the doctrine of Fourier transformation. According to human electroencephalography nomenclature, four different EEG rhythms may be distinguished, namely beta, alpha, theta, and delta. Beta activity is a frequency wave higher than $12.1 \mathrm{~Hz}$, and its presence is highly dependent on normal cortical function. It is seen in the EEG of normal people who are awake with eyes open $(12,41)$. Alpha activity is a frequency wave between 8.1 and $12 \mathrm{~Hz}$ and amplitude of $20-60 \mu \mathrm{V}$, which is evident in normal people who are awake with eyes closed, but disappears in drowsiness and sleep. This rhythm is usually observed in the posterior area (12). Theta rhythm is a frequency wave between $4.1-8.0 \mathrm{~Hz}$ that appears in the second, third, and fourth stages of physiological non-rapid eye movement (NREM) sleep, but it can also be observed in different pathological states. Delta wave is characterised by a frequency of $0.5-4.0 \mathrm{~Hz}$, and is seen in EEG in the third and fourth stages of NREM sleep (12). Theta and delta activities were defined as "high voltage low frequency" visible in the EEG pattern for drowsiness and sleep (12).

The recording analysis is usually performed on a monopolar and/or bipolar montage modality (Fig. 4). Physiological and pathological transients are marked and recorded on a recording event list (Fig. 4). Special emphasis should be placed on detection of artefact, including: ocular movements, cardiovascular and muscular activity, physiological rhythmic movements, or recording environmental artefacts. All should be identified and differentiated from the pathological SITs (e.g. muscular artefact from spike activity) by the examining person and properly interpreted. The spectral bands in q-EEG are defined as delta for the 
frequency values of $0.5-4.0 \mathrm{~Hz}$, theta for $4.1-8.0 \mathrm{~Hz}$, alpha for $8.1-12.0 \mathrm{~Hz}$, and beta for the values between 12.1 and $30.0 \mathrm{~Hz}$.

Current applications of the EEG in dogs. There is a list of important indications for EEG use in human medicine including: assistance in diagnosis and classification of seizure disorders, confirmation of encephalopathy by diffuse slowing of EEG rhythms, localisation of functional lesions when imaging studies are negative, diagnosis of sleep disorders, confirmation of brain death, prediction of prognosis in comatose patients (7). The use of EEG enables identification of a few "signature" disorders: herpes simplex encephalitis (PLEDs), Creutzfeld-Jakob disease (periodic frontal waves), hepatic encephalopathy (triphasics), subacute sclerosing panencephalitis (41).
The most important applications of EEG use in dogs include: detection of the interictal epileptic discharges (IED), EEG monitoring of the status epilepticus, differentiation of epileptic and nonepileptic disorders, diagnosis of metabolic encephalopathies, and corticogram.

Detection of the interictal epileptic discharges (IED) for seizure diagnosis. The terminology of investigated interictal epileptic discharges (IED) used in veterinary medicine is adapted from currently accepted basic terminology of the International Federation of the Society for Electroencephalography and Clinical Neurology (31). A spike is defined as "transient, clearly distinguished from the BGA, with pointed peak at conventional paper speed and a duration from 20 to under $70 \mathrm{~ms}$; the main component is generally negative. Amplitude is variable".

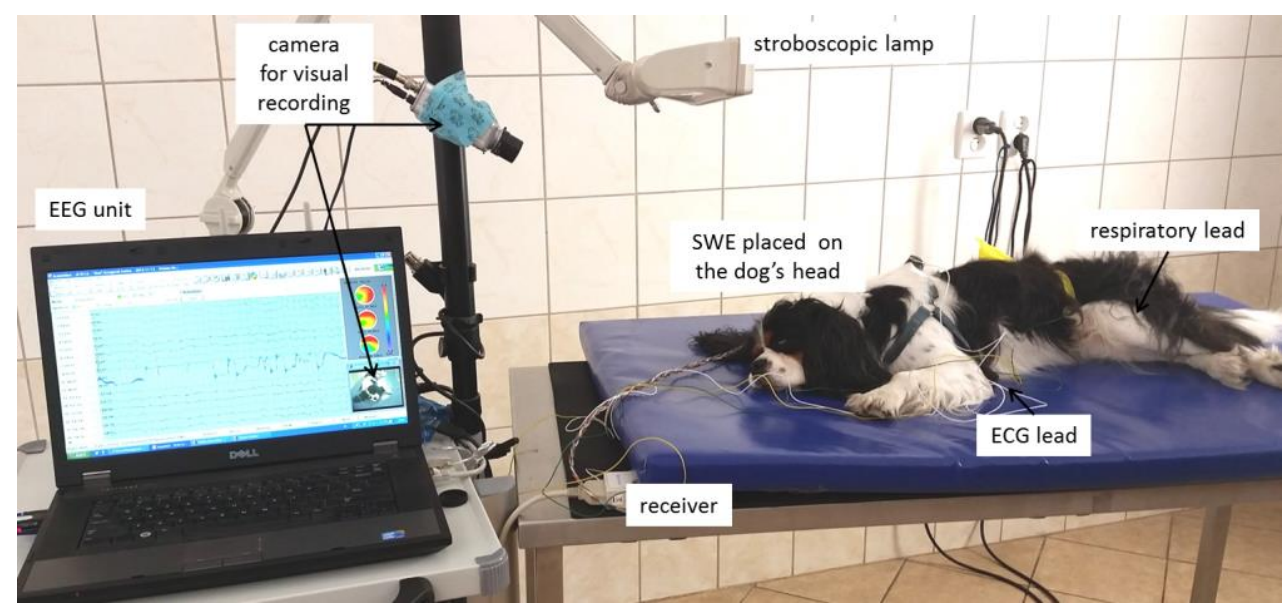

Fig. 3. An example electroencephalographic set for canine EEG recording with videometry. The examined dog in sedation is placed in sternal recumbency. During the EEC acquisition the examination room should be darkened

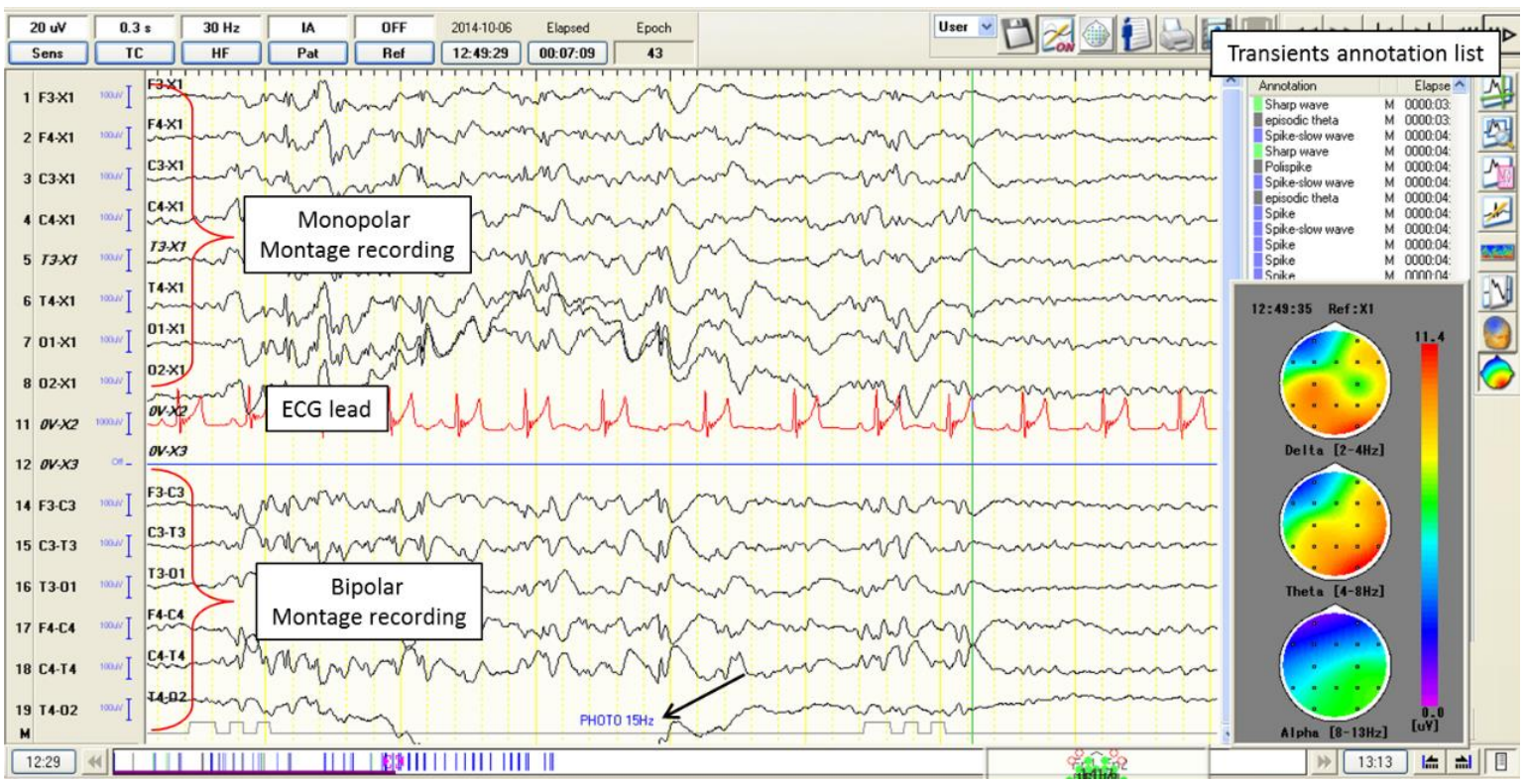

Fig. 4. A canine EEG 8-channel recording for review. The simultaneous mono- and bipolar montages are presented. An ECG recording simultaneously viewed for possible artefact detection. The transient annotation list for defined superimposed transients (SITs) showed Two background activities visible (BGA), before the green line high-voltage, low frequency (HVLF), and after the green line low-voltage, high frequency (LVHF). The recording performed during the photic stimulation (arrow indicates the stimulation of $15 \mathrm{~Hz}$ ). $(\mathrm{F}$ - frontal; $\mathrm{T}$-temporal; $\mathrm{C}$ - central; $\mathrm{O}$ - occipital; odd numbers = left hemisphere; even numbers = right hemisphere $)$ 


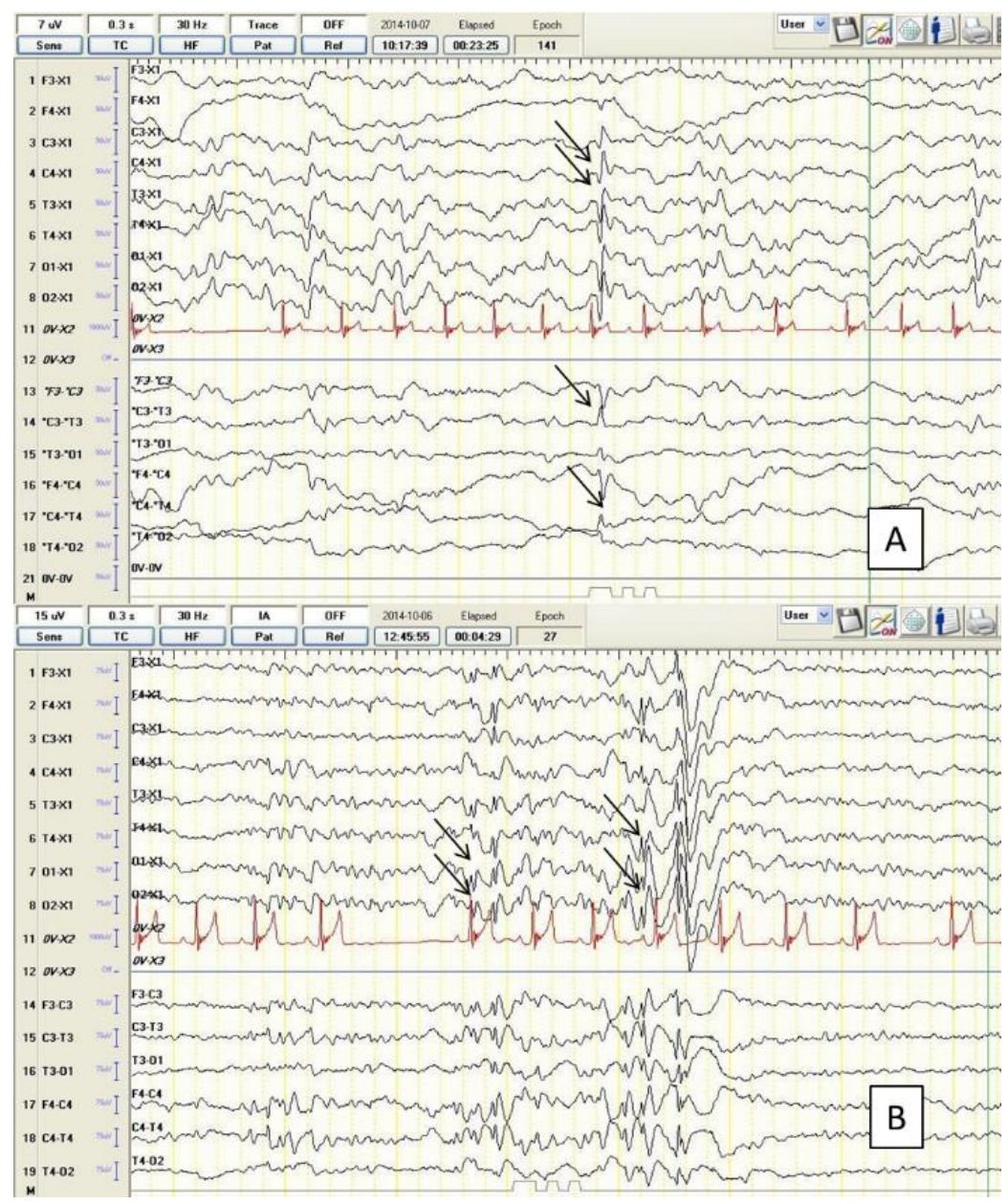

Fig. 5. The examples of pathological superimposed transients (SITs) of interictal epileptic discharges (IED) seen in canine epilepsy patients. A. Spike activity (arrows) in C3, C4 leads. In monopolar montage the amplitudes in $\mathrm{C} 3, \mathrm{C} 4$ are the highest, in bipolar a reversed polarity is seen (spikes directed towards each other, (arrows)). B. Polyspikes activity (arrows) significantly stand out from the background activity (BGA)

A sharp wave is defined as "a transient wave, clearly distinguished from the BGA, with a pointed peak at conventional paper speed and a duration from 70 to $200 \mathrm{~ms}$; the main component is generally negative relative to the areas" and a spike wavecomplex is defined as "a pattern consisting of a spike followed by slow wave". Polispikes and poli-sharp waves are graphoelement that are combined of multiple spikes and sharp waves (Fig. 5). The IED detection rate in dogs was variously estimated by different researches, starting from the lowest values of $12.5 \%$ (34), $20 \%-29 \%(23,39,7), 55 \%-65 \%(36,16,2)$, up to $86 \%-100 \%(19,26,30)$. The detection rate seems to be related to the sedation and montage usage.

The use of EEG in monitoring status epilepticus. The use of continuous EEG recording was described in a retrospective study in order to assess 10 dogs with status epilepticus (37). Animals were treated with diazepam and pentobarbital in order to stop the seizures. In all cases clinical seizures were diminished, but EEG recording still showed spikes and polispikes in all dogs. Those paroxysms were suppressed by increasing the infusion rate of anaesthetics. In this study EEG was found to be useful for monitoring the cortical activity during status epilepticus and significantly influenced the treatment protocol. The continuous EEG (cEEG) is used in humans in intensive care units (14), but this technique is not routinely used in veterinary medicine.

Differentiation of epileptic and non-epileptic disorders. The EEG examination of 24 dogs with flycatching syndrome (FCS) from three different neurological centres revealed epileptic discharges (ED) of spikes detected in $8 / 21$ (38\%) cases. In this group, $36 \%$ of the dogs responded to phenobarbital and $100 \%$ to fluoxetine, which together with EEG examination results could have suggested that the FCS is not a form of seizure, but another disorder, such as obsessivecompulsive (OCD) (46). The EEG examination in this report was found to be not specific enough for the differentiation of the two conditions (seizures vs. OCD) in dogs.

The use of EEG in metabolic encephalopathies. In the study on 12 dogs with hepatic encephalopathy (porto-systemic shunt encephalopathy), EEG examination revealed BGA of high voltage, low- 
frequency (HVLF), with the presence of pathological SITs of bilateral symmetric triphasic waves in $58 \%$ of the cases and spread bisynchronous and asynchronous slow waves in $83 \%$ (45). Both SITs seem to be characteristic for the metabolic encephalopathies in humans (25).

Corticogram, experimental use of EEG in dogs. A study on six dogs was performed with the use of intracranial EEG (iEEG) with 16 channels (10). Out of the total of $11,671 \mathrm{~h}$ of EEG recording, only $3.8 \%$ were not readable due to interruption in the transmitted signal. A total of 202 detected electrographic seizures were identified and $91.1 \%$ were focal. The device platform presented in this study has a great potential to impact epilepsy research, with a canine epilepsy model suitable for humans. However, high invasiveness does not allow using this technique as a standard protocol for dogs as veterinary outpatients.

Polymicrogyria in poodles. In a study describing an anomaly of polymicrogyria in poodles, EEG was performed in one of five dogs. The electroencephalogram showed spike-wave discharges with phase reversal over the central derivations. In this study, dogs were sedated with acepromazine and butorphanol. In humans with this disease, up to $41.5 \%$ $55 \%$ have normal EEG and the changes found are not disease-specific (43). In the presented case, the occurrence of IED increased the suspicion that untypical behaviour of circling and lip smacking were likely to be complex partial motor seizures (24).

In conclusion, EEG examination is part of the routine diagnostic work-up in human medicine. The interest of veterinary scientists in EEG increases, due to the development of large medical data of computed analysis techniques (software), which improve EEG recording analysis and interpretation. The potential use of EEG in canine patients includes precise detection of the cortical functional disturbances, such as pathological interictal epileptiform discharges (IEDs) and its exact cortical (or subcortical) mapping for the possible neurosurgical treatment of seizures in dogs. EEG in canine patients could be also used for epilepsy treatment monitoring in order to select the most optimal anti-epileptic drugs. The technique could also allow differentiation of the non-epileptic and epileptic disorders (head tremors, ticks, various abnormal behavioural disturbances, or involuntary movement differentiation from seizures), that could significantly influence the choice of treatment approach.

Conflict of Interests Statement: The author declares that there is no conflict of interests regarding the publication of this article.

Acknowledgements: The support of the Faculty of Veterinary Medicine, Wroclaw University of Environmental and Life Sciences, Poland, under its statutory activity is gratefully acknowledged.

\section{References}

1. Arain A.M., Arbogast P.G., Abou-Khalil B.W.: Utility of daily supervised hyperventilation during long-term video-EEG monitoring. J Clin Neurophysiol 2009, 26, 17-20.

2. Berendt M., Hogenhaven H., Flagstad A., Dam A.: Electroencephalography in dogs with epilepsy: similarities between human and canine findings. Acta Neurol Scand 1999, 99, 276-283.

3. Bergamasco L., Accatino A., Jaggy A.: Methodical approach to digital electroencephalography and its use in veterinary medicine. Veterinaria 1999, 13, 7-22.

4. Bergamasco L., Accatino A., Priano L., Neiger-Aeschbacher G., Cizinauskas S., Jaggy A.: Quantitative electroencephalographic findings in beagles anaesthetized with propofol. Vet J 2003, 166, 58-66.

5. Berger H.: Über das Elektrenkephalogramm des Menschen. Arch Psychiatr Nervenkr 1929, 87, 527-570.

6. Brauer C., Kastner S.B., Rohn K., Schenk H.C., Tunsmeyer J., Tipold A.: Electroencephalographic recordings in dogs suffering from idiopathic and symptomatic epilepsy: diagnostic value of interictal short time EEG protocols supplemented by two activation techniques. Vet J 2012, 193, 185-192.

7. Brauer C., Kastner S.B., Schenk H.C., Tunsmeyer J., Tipold A.: Electroencephalographic recordings in dogs: Prevention of muscle artifacts and evaluation of two activation techniques in healthy individuals. Res Vet Sci 2011, 90, 306-311.

8. Croft P.G.: The EEG as an aid to diagnosis of nervous diseases in the dog and cat. J Small Anim Pract 1962, 3, 205-213.

9. Croft P.G.: Canine Epilepsy. Vet Rec 1988, 122, 238-239.

10. Davis K.A., Sturges B.K., Vite C.H., Ruedebusch V., Worrell G., Gardner A.B., Leyde K., Sheffield W.D., Litt B.: A novel implanted device to wirelessly record and analyze continuous intracranial canine EEG. Epilepsy Res 2011, 96, $116-122$.

11. Doose H., Waltz, S.: Photosensitivity-genetics and clinical significance. Neuropediatrics 1993, 24, 249-255.

12. Fisch B.J.: Fisch and Spehlmann's EEG primer. Basic principles of digital and analog EEG. Amsterdam, Elsevier B.V., 1999, pp. 126, 185-195, 199-206, 379.

13. Flink R., Pedersen B., Guekht A.B., Malmgren K., Michelucci R., Neville B., Pinto F., Stephani U., Ozkara C.: Guidelines for the use of EEG methodology in the diagnosis of epilepsy. Acta Neurol Scand 2002, 106, 1-7.

14. Friedman D., Claassen J., Hirsch L.J.: Continuous electroencephalogram monitoring in the intensive care unit. Anesth Analg 2009, 109, 506-523.

15. Herin R.A., Purinton P.T., Fletcher T.F.: Electroencephalography in the unanesthetized dog. Am J Vet Res. 1968, 29, 329-336.

16. Holliday T.A., Cunningham J.G., Gutnick M.J.: Comparative clinical and electroencephalographic studies of canine epilepsy. Epilepsia 1970, 11, 281-292.

17. Holliday T.A., Williams D.C.: Interictal paroxysmal discharges in the electroencephalograms of epileptic dogs. Clin Tech Small Anim Pract 1998, 13, 132-143.

18. Itamoto K., Taura Y., Wada N., Taga A., Takuma T., Matsumura H., Miyara T.: Effect of medetomidine on electroencephalography and use of a quantitative electroencephalograph for evaluating sedation levels in dogs. J Vet Med A Physiol Pathol Clin Med 2001, 48, 525-535.

19. Jaggy A., Bernardini M.: Idiopathic epilepsy in 125 dogs: a longterm study. Clinical and electroencephalographic findings. J Small Anim Pract 1998, 39, 23-29.

20. Jallon P.: Electroencephalogram and epilepsy. Eur Neurol 1994, 34 (Suppl. 1), 18-23.

21. James F.M., Allen D.G., Bersenas A.M., Grovum W.L., Kerr C.L., Monteith G., Parent J.M., Poma R.: Investigation of the use of three electroencephalographic electrodes for long-term electroencephalographic recording in awake and sedated dogs. Am J Vet Res 2011, 72, 384-390. 
22. Jeavons P. M., Bishop, A., Harding, G. F.: The prognosis of photosensitivity. Epilepsia 1986, 27, 569-575.

23. Jeserevics J., Viitmaa R., Cizinauskas S., Sainio K., Jokinen T.S., Snellman M., Bellino C., Bergamasco L.: Electroencephalography findings in healthy and Finnish Spitz dogs with epilepsy: visual and background quantitative analysis. J Vet Intern Med 2007, 21, 1299-1306.

24. Jurney C., Haddad J., Crawford N., Miller A.D., Van Winkle T.J., Vite C.H., Sponenberg P., Inzana K.D., Cook C.R., Britt L., O'Brien D.P.: Polymicrogyria in standard poodles. J Vet Intern Med. 2009, 23, 871-874.

25. Kaplan P.W., Rossetti A.O.: EEG patterns and imaging correlations in encephalopathy: encephalopathy part II. J Clin Neurophysiol 2011, 28, 233-251.

26. Klemm W.R., Hall C.L.: Electroencephalographic "seizures" in anesthetized dogs with neurologic diseases. J Am Vet Med Assoc 1970, 157, 1640-1655.

27. Klemm W.R.: Electroencephalograms of anesthetized dogs and cats with neurological diseases. Am J Vet Res 1968, 29, 337-351.

28. Koutroumanidis M., Smith S.: Use and abuse of EEG in the diagnosis of idiopathic generalized epilepsies. Epilepsia 2005, 46 (Suppl 9), 96-107.

29. Mirsattari S.M, Bihari F., Leung L.S., Menon R.S., Wang Z., Ives J.R., Bartha R.: Electroencephalographic recording during transcranial magnetic stimulation in humans and animals. Clin Neurophysiol 2005, 144, 207-213.

30. Morita T., Shimada A., Takeuchi T., Hikasa Y., Sawada M., Ohiwa S., Takahashi M., Kubo N., Shibahara T., Miyata H., Ohama E.: Cliniconeuropathologic findings of familial frontal lobe epilepsy in Shetland sheepdogs. Can J Vet Res 2002, 66, 35-41.

31. Nordli D.R., Riviello J., Niedermeyer E.: Seizures and epilepsy in infants to adolescents. In: Niedermeyer's Electroencephalography. Basic Principles, Clinical Applications, and Related Fields. Edited by Schomer D.L., Da Silva F.L., Lippincott, Philadelphia, 2011, pp. 479-540.

32. Otto K., Short C.E.: Electroencephalographic power spectrum analysis as a monitor of anesthetic depth in horses. Vet Surg 1991, 20, 362-371.

33. Packer R.M.A., Berendt M., Bhatti S., Charalambous M., Cizinauskas S., De Riso L., Farquhar R., Hampel R., Hill M., Mandigers P.J., Pakozdy A., Preston S.M., Rusbridge C., Stein V.M., Taylor-Brown F., Tipold A., Volk H.A.: Interobserver agreement of canine and feline paroxysmal event semiology and classification by veterinary neurology specialists and non-specialists. BMC Vet Res 2015, 11, 39

34. Pakozdy A., Thalhammer J.G., Leschnik M., Halasz P.: Electroencephalographic examination of epileptic dogs under propofol restraint. Acta Vet Hung 2012, 60, 309-324.

35. Patel V.M., Maulsby R.L.: How hyperventilation alters the electroencephalogram: a review of controversial viewpoints emphasizing neurophysiological mechanisms. J Clin Neurophysiol 1987, 4, 101-120.

36. Pellegrino F.C., Sica R.E.: Canine electroencephalographic recording technique: findings in normal and epileptic dogs. Clin Neurophysiol 2004, 115, 477-487.

37. Raith K., Steinberg T., Fischer A.: Continuous electroencephalographic monitoring of status epilepticus in dogs and cats: 10 patients (2004-2005). J Vet Emerg Crit Care 2010, 20, 446-455.

38. Redding R.W, Colwell R.K.: Verification of the significance of the canine electroencephalogram by comparison with the electrocorticogram. Am J Vet Res 1964, 25, 857-861.

39. Redding R.W.: Atlas of Electroencephalography in the Dog and Cat. Praeger Publisher, New York, 1984.

40. Sim M.K., Chua M.E.: EEG in anaesthetized rats. Pharmacol Toxicol 1989, 65, 119-120.

41. Smith S.J.: EEG in the diagnosis, classification, and management of patients with epilepsy. J Neurol Neurosurg Psychiatry 2005, 76 (Suppl 2), 2-7.

42. Striano S., Meo R., Bilo L., Ruosi P., Soricellis M., Estraneo A., Caporella A.: The use of EEG activating procedures in epileptology. Acta Neurol 1992, 14, 275-289.

43. Teizeira K.C.S., Montenegro M.A., Cendes F., Guimaraes C.A., Guerreiro C.A., Guerreiro M.M.: Clinical and electroencephalographic features of patients with polymicrogyria. J Clin Neurophysiol 2007, 24, 244-251.

44. Wrzosek M., Nicpon J., Bergamasco L., Sammartano F., Cizinauskas S., Jaggy A.: Visual and quantitative electroencephalographic analysis of healthy young and adult cats under medetomidine sedation. Vet J 2009, 180, 221-230.

45. Wrzosek M., Płonek M., Nicpoń J.: Electroencephalographic features of metabolic encephalopathy in dogs. Med Weter 2015, 71, 100-103.

46. Wrzosek M., Płonek M., Nicpoń J., Cizinauskas S., Pakozdy A.: Retrospective multicenter evaluation of the "fly-catching syndrome" in 24 dogs: EEG, BAER, MRI, CSF findings and response to anti-epileptic and anti-depressant treatment. Epilepsy Behav 2015, 53, 184-189. 